\title{
Smart Body Posture Guidance System
}

\author{
Anitha.J, Dhivya. D, Harishma.S, Mythili.N
}

\begin{abstract}
Body posture plays a crucial role in the measurement of body vitals. Accurate measurement of blood pressure, body weight, etc. requires correct posture which greatly affects the result. To achieve the correct posture the person has to depend on the lab technician, who helps the person to sit in the correct pose, to place the cuff in the right place to measure blood pressure, to stand in the correct pose to measure body weight, etc. To eliminate the need for lab technician, we develop a system, to capture image and recognize the pose using a camera, image processing using pose estimation algorithm, guide the person to correct if the recognized pose is incorrect using a User Interface developed using pyqt. The pose estimation algorithm works by estimating and connecting the body keypoints. Hence we are creating a prototype model for real time pose estimation and guidance.
\end{abstract} Kiosk.

Key terms: Body Keypoints, Deep Learning, GUI, Health

\section{INTRODUCTION}

Smart body posture guidance system recognizes the human body by estimating the body keypoints, based on deep learning. Real time depth cameras have made this task easier. The main aim of the system is to eliminate the need for lab technician while checking body vitals like body weight, blood pressure, etc. Here, we use image processing techniques to estimate the pose and compare it with the guidance images.. The system makes use of images captured by the camera which is integrated to the system.Body point based posture analysis is the state of art for posture recognition. Deep learning models trained on key point estimation are used for understanding the complex poses exhibited during different activities with ease. These models are trained on 3-D triangulation from multiple single views, there enabling them the capability to handle occlusion. The correct body posture is essential in accurate estimation of body vitals like BP measurement. The person using the system will be alerted with the help of guidance images in case of incorrect posture has been detected. In recent years, with the rapid development of virtual reality, augmented reality, and interactive multimedia, human-computer interaction (HCI) technology is in higher demand than ever before.

\section{Components of proposed system}

\section{Camera}

The specification of the required camera is C922 pro stream web cam. The camera is the first and foremost requirement of the system since it provides the input.

Revised Manuscript Received on June 30, 2020.

* Correspondence Author

Dr. J. Anitha*, Associate Professor, Department of Information Technology, Sri Ramakrishna Engineering College, Coimbatore, India.

D. Dhivya, Department of Information Technology, Sri Ramakrishna Engineering College, Coimbatore, India.

S. Harishma, Department of Information Technology, Sri Ramakrishna Engineering College, Coimbatore, India.

N. Mythili, Department of Information Technology, Sri Ramakrishna Engineering College, Coimbatore, India.

(C) The Authors. Published by Blue Eyes Intelligence Engineering and Sciences Publication (BEIESP). This is an open access article under the CC BY-NC-ND license (http://creativecommons.org/licenses/by-nc-nd/4.0/)
The high resolution camera enables the system to capture high quality image which will be helpful to recognize the posture and will also be useful to guide the user of the system.

PC

The PC used in this system is with i5 processor, which is linked to the camera. The captured image and the guidance image will be displayed in the PC.

\section{RELATED WORK}

Smart body posture guidance system is mainly developed to sit in a right posture while measuring blood pressure, which plays a vital role in the accuracy of the result, without the help of lab technician. We also need exposure to pose estimation.

M. Andriluka [1] developed a 3-D pose estimation system in three stages to estimate and track multiple people in crowded areas using potentially moving cameras, to overcome the inherent ambiguity and complexity of human articulation.

Demirdjian [2] presented progress on untethered articulation of arm and body. Virtual reality based sensors are used for tracking body configuration. This system allows tracking of real time, robust and accurate tracking of 3-D position and tracking.

P.F.Felzenszwalb [3] presented a framework for object recognition. Each part of the object is modelled separately and allows qualitative descriptions of visual appearance. The problem of using pictorial models and learning from training examples are addressed.

Ashwini Magar [4] developed a system which uses haar cascades to segment human from images eliminating the background. It faces large variability of shapes, appearance of object class. Using haar cascades technology the input image will be divided into upper and lower body so that it will be easier to segment the human image alone.

Abhishek Yadav [5] developed a system which uses head as the key for body-pose estimation. Artificial neural networks are trained to obtain the head position. Matlab platform is used to develop the system. This system is developed to recognize the activity and name the exercise performed by the person.

\section{PROPOSED METHODOLOGY}

Smart body posture guidance system is developed to eliminate the need for lab technician to help us while measuring blood pressure, weight, etc. This system is to detect the posture in real time and helps to correct the posture which will be helpful to obtain the accurate measurement.

Visual studio

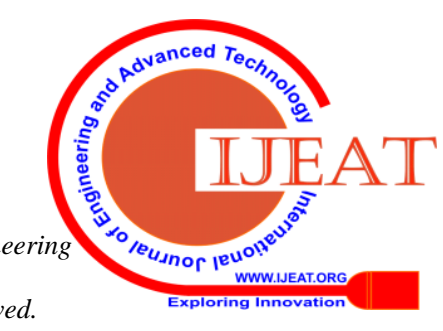




\section{Smart Body Posture Guidance System}

Visual studio is used to develop programs, which supports 36 different languages. The layout developed using visual studio can be controlled housing the controls inside other containers or locking them to the side of the form. The UI is linked with code using an event driven programming model.

\section{PyQt 5}

PyQt 5 is the tool which is used for development of Graphical User Interface. It is a high performance GUI development platform implemented in python. Since it offers wide range of menus, buttons, it is widely chosen over many alternatives and also permits code flexibility. The user interface of the system has been developed using Pyqt5 which would enable the patients to know their status by displaying the captured image on the screen. The interface screen also displays the guidance images which guides the patient to sit in the correct posture. The system also enables the patient to start or stop the process. The flags in the GUI indicate the mistake by turning red while green indicates correct posture.

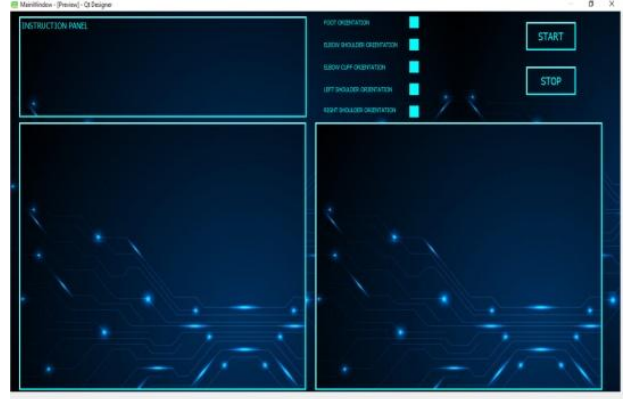

Fig.1 .User Interface

\section{Workflow of proposed system}

The workflow of Smart body posture guidance system involves the following steps:

Step 1: The camera interfaced with the system captures the image of the person sitting in front of the system.

Step 2: The captured image is displayed in the GUI.

Step 3: The model weight will be downloaded and then load network.

Step 4: Read the captured image as input and prepare input to the network .To make predictions and parse

key points.

Step 5: The image display with key points after running pose estimation algorithm.

Step 6: If the person is found to be sitting in the wrong posture, indicators will alert the person and make them to correct

Step 7: The GUI also displays the correct postures which will be helpful to guide the person

\section{RESULTS AND OUTPUT}

The real-time video image is acquired by using the deep learning network model completed by training, and the pose characteristics of each frame are extracted, and then the correctness of the sitting posture is judged. The recognition results are printed in the video interface, as shown in Figures 2, 3 and 4 each of picture is a screenshot of the video display interface. The output will show the GUI display which has three sections. The first section shows instructions and instruction images to patients .The section indicates orientation of image is taken by camera and is processed in model using green and red as sign where green indicates correct posture and red indicates wrong posture. The third section indicates image taken by camera using key points.

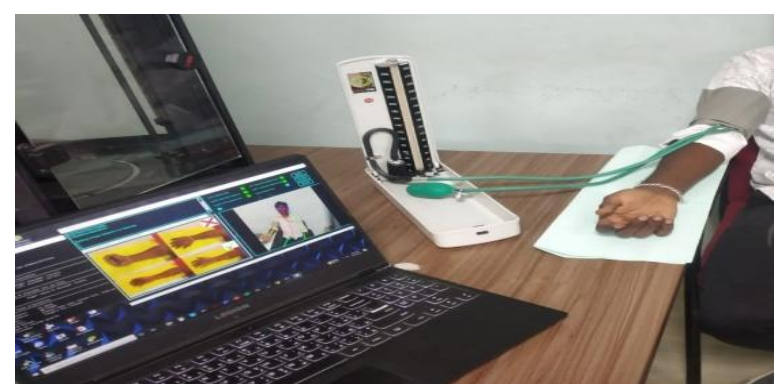

Fig. 2.Hardware set up

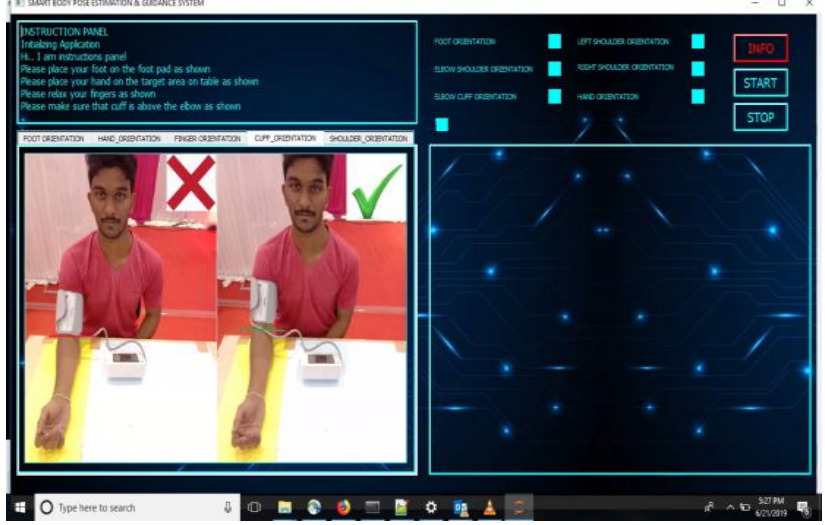

Fig.3.Output of guidance system

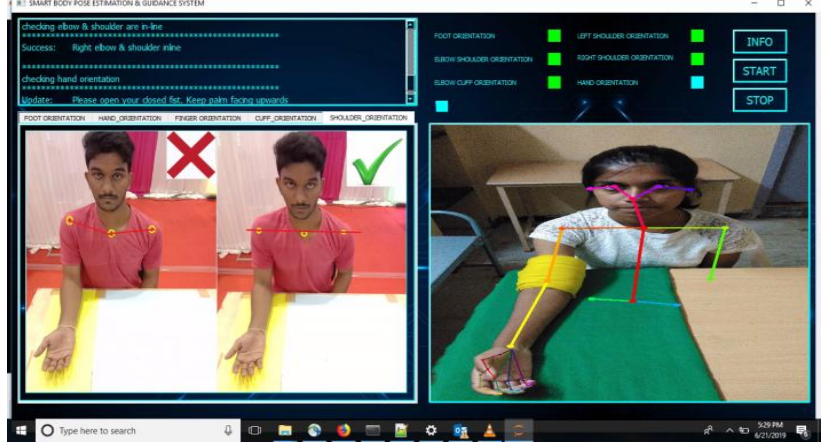

Fig .4 Final output

\section{CONCLUSION}

Hence we are developing a prototype model to prevent the person from sitting in incorrect posture by alerting them using the GUI. The camera captures the image of the person using this system, and by using image processing and pose estimation algorithm we can estimate the posture. So that there will not be a need for lab technician to help the patients. In future it can be extended to use in IT industries to monitor the prolonged pose of the professionals.

\section{REFERENCES}

1. M. Andriluka, S. Roth, and B. Schiele(2010) Monocular 3D pose estimation and tracking by detection-CVPR.

2. Demirdjian(2002)-3-DArticulatedPose Tracking for Untethered Diectic Reference in the proceedings of IEEE Multimodal interfaces, pp. 267-272.

Published By:

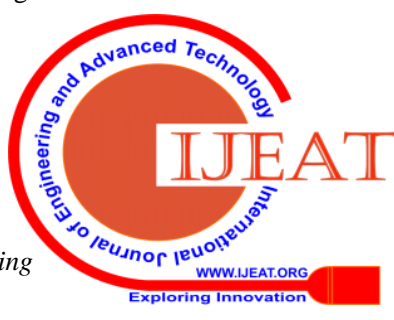

Blue Eyes Intelligence Engineering

\& Sciences Publication

(c) Copyright: All rights reserved. 
3. P.F.Felzenszwalb and D.P. Huttenlocher, (2005) -Pictorial structures for object recognition, International Journal of computer vision, Vol.61, pp. 55-79.

4. Ashwini Magar, Prof.J.V.Shinde,(2015)-A New Approach of Human Segmentation from Photo Images ,International Journal of Scientific and Research Publications,pp 1045-1053.

5. Abhishek Yadav and Vijay Kumar Joshi, (2017)-Human Body Detection with Segmentation Using Spline Regression, International Journal of Informative \& Futuristic Research, pp. 7440-7449

6. P. Lokeshwara Reddy, R. V. Sree Hari, (2017)-Extraction of Standing Human Bodies from Images using Multi Level Segmentation, International Journal of Scientific Engineering and Technology Research, pp. 5208-5213.

7. Misra, A. Shrivastava, A. Gupta, and M. Hebert,(2016) -Cross-stitch networks for multi-task learning, IEEE Conference on Computer Vision and Pattern Recognition, pp. 3994-4003.

8. Prathik Chattopadhyay , Shamik Sural , Jayanta Mukherjee,(2014)Frontal Gait Recognition from Incomplete Sequences using RGB-D Camera, IEEE transaction on information forensis and security, pp.1843-1846, vol.9(11).

9. L. Sigal and M. J. Black, (2006)-Measure locally, reason globally: Occlusion-sensitive articulated pose estimation,IEEE Computer Society conference on computer vision and pattern recognition (CVPR'06),pp 123-145.

10. Khamparia, A and Pandey, B,(2018)- SVM and PCA based learning feature classification approaches for E-learning system, International Journal of Web-based Learning Performance in Defense-Based Enhanced Learning, Vol. 9, No. 1, pp.37- 50.

\section{AUTHORS PROFILE}

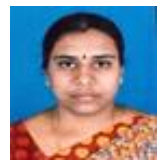

Dr.J.Anitha is currently working as Associate Professor in the Department of Information Technology at Sri Ramakrishna Engineering College, Coimbatore. She has 17 years of teaching and industrial experience.

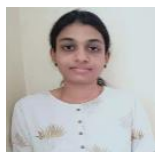

D.Dhivya is currently studying B.Tech in Information Technology at Sri Ramakrishna Engineering College in Coimbatore, India.

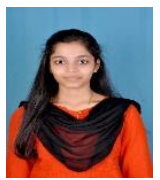

S.Harishma is currently studying B.Tech in Information Technology at Sri Ramakrishna Engineering College in Coimbatore, India.

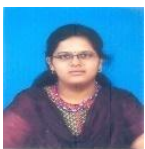

N.Mythili is currently studying B.Tech in Information Technology at Sri Ramakrishna Engineering College in Coimbatore, India

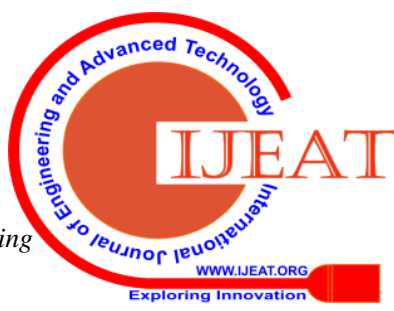

